\title{
The Manuscripts and Editorial Principles
}

The manuscripts that we have transcribed and publish in this volume belong to the Special Collections Centre in the Sir Duncan Rice Library, University of Aberdeen. Most of the manuscripts are part of the Birkwood Collection, while the remainder are found in a significant collection of Reid's papers given to the Library in 1980. Details of the physical state of each manuscript and authorial revisions and annotations are given in the Textual Notes. All of the manuscripts in the Birkwood Collection have been digitised and are available on the website of the Library (http://www.abdn.ac.uk/diss/historic/Thomas_Reid).

It appears that Reid himself organised his papers thematically, for among the manuscripts now located in the bundle MS 2131/4/III/12-23c there is an uncatalogued wrapper labelled 'Politicks' in Reid's hand. This wrapper was used to keep together the papers related to Reid's politics lectures now found in the MS 2131/4/ III/1-23c sequence. After Reid's death, his colleague George Jardine arranged all of the surviving manuscripts into ten topical groups, and the wrappers for these ten parcels are now kept in the box containing the manuscripts catalogued as MS 2131/8/I-VII. The wrappers themselves are uncatalogued. A transcription of them will be published in volume 10 of the Edinburgh Edition of Thomas Reid. In addition to the original wrappers there is an equivalent set created in the nineteenth or twentieth century. This second set of wrappers has labels written in ink that are transcriptions of Jardine's labels affixed to the original wrappers. Despite these efforts to arrange the manuscripts into topical groups, the thematic ordering of Reid's papers has in fact been significantly disturbed at various points, so that their catalogued order in many cases bears little relationship to their original intellectual order. Moreover, Reid himself reworked material with revisions, deletions, insertions and additions written in margins or inter-lined in the original text. He also used blank parts of paper for miscellaneous notes of little or no relevance to the original text of the document. His writing paper was typically a sheet that was folded and refolded to form an approximately octavo-sized quire of four leaves held together with a small string (which often has broken, leaving the leaves free to migrate elsewhere or be lost entirely). All these factors conspire to make our reconstruction of Reid's lectures on politics subject to some uncertainty 
and to leave several gaps in it. We can only confirm what George Jardine wrote on the label of the relevant bundle (Wrapper B):

This Parcel Contains Heads of Lectures on Pneumatology, Ethicks \& Politicks which seem to have been delivered the first Session Dr. Reid Taught in Glasgow College. To which so many additional Notes \& Illustrations have been made in Succeeding years that they cannot easily be put in exact order.

The papers that Reid presented to the Aberdeen Philosophical Society and the Glasgow Literary Society, included in Part Three of this volume, pose less of an editorial challenge than his lecture notes that make up Part Two. While not in all cases without problems for modern editors, these papers were sufficiently polished to be read aloud. The reading notes transcribed in Part One are the least problematic from an editorial point of view, even though they sometimes raise serious issues of interpretation.

Our aim in transcribing the manuscripts included in this volume has been to provide accurate and easily readable texts, while preserving the integrity of Reid's writing. Because most of the manuscripts contain numerous deletions, insertions, corrections and the like, the creation of such texts is not a straightforward process. We have therefore adopted the following principles in order to achieve our editorial end:

1. We have made no attempt to normalise Reid's erratic spelling and punctuation, except where the spelling was clearly mistaken or its eccentricity too distracting.

2. In our transcriptions, words or characters which are missing because of damage to the manuscript or are judged to have been inadvertently omitted by Reid are enclosed thus: ' $\langle$ '. Illegible letters or words are indicated thus: ' $\langle$ ?'.

3. Any characters written as superscripts are here printed not raised.

4. In Reid's manuscripts, he typically overlines for emphasis, and we have reproduced the relevant words or passages in italics without editorial comment.

5. We have silently normalised Reid's contractions and abbreviations where no modern equivalent exists, or where they are not self-explanatory or readily pronounceable by the modern reader. Thus, on page 3, line 1 , we have not expanded 'Aug' because it is still recognised as an abbreviation for 'August', but on the next line we have normalised Reid's contraction 'Cha' because it does not obviously stand for 'Charles'. We have also silently expanded some of Reid's contractions in the interests of readability. For example, on page 3 , line 6 , we have normalised Reid's 'Art' to 'Article' because it occurs in a reading context. But where contractions occur in the context of a reference (as on page 10, line 1), we 
have usually left them unexpanded because their meaning will become obvious from the information supplied in our explanatory notes.

6. We have silently omitted repetitions of words or phrases, catchwords and any material deemed to have been mistakenly included in the manuscripts.

7. Folio/page breaks are indicated by a vertical line, 'l', in the text. In the page margin at the line in which Reid began a new folio or page, the manuscript number is printed together with the folio or page number. There are two types of manuscript numbers in the volume, respectively of these forms: $2131 / 2 / \mathrm{II} / 14$ and $3061 / 3$. The former type always has the first four digits (2131) in common and these are left out in the marginal printing. The manuscript number is followed, after a comma, by an indication of the folio or page number in the original manuscript; in the case of unpaginated manuscripts, we give the folio number and the side - recto ' $r$ ' or verso ' $v$ ' - of the folio. The folio/page indications should allow the reader to follow the way in which we have arranged the manuscripts that remain from Reid's lectures on politics. As mentioned, Reid often used the same sheet of paper for different purposes, and it has therefore been necessary to divide a number of manuscripts. Conversely, the rejoining of manuscripts that cohered originally has meant that their folios/pages are printed here in an order different from that in which they happen to have been preserved.

8. In the transcriptions of Reid's manuscripts we have added line numbers. These are used for reference to the Textual and Editorial Notes (see points 9 and 10 below). In cases where the line numbers coincide with the manuscript numbers (cf. point 7 above), the line number is not included.

9. Variants in Reid's manuscripts are recorded in the Textual Notes, and these are keyed to the relevant texts using page and line numbers (that is, ' $18 / 32$ ' in the Textual Notes refers to p. 18, 1. 32 in this volume). In these notes, editorial comment is in italics and Reid's wordings are in roman typeface. Instances where Reid has changed an unfinished text by superimposing a letter or word over what he had originally written, or revised a phrase in the course of his initial writing, have been used in our transcriptions but have not been recorded in the Textual Notes. We have also not recorded those instances where Reid has merely gone back and corrected his spelling or grammar, or supplied a missing character or word. Variants are indicated in the following manner. In MS 2131/4/III/3 (p. 32, 1. 13 below), for example, Reid initially wrote 'something in the Nature of it', then deleted 'the Nature of', leaving as his final formulation 'something in it'. Reid's change is recorded in the Textual Notes thus: something in it] something in the Nature of it.

Reid often failed to replace deleted material with a new word or phrase. These cancellations have been identified and recorded in the Textual Notes. 
In recording Reid's revisions we have made a distinction between insertions and additions. Where Reid has indicated where to place additional material in the text (typically with a '^'symbol below the line) we have used the annotation 'inserted'. Where Reid has not indicated where to place additional material with a symbol, we have used the annotation 'added'.

In ambiguous cases we have specified the revision using the normal convention illustrated above. Where Reid has written his insertion or addition in the margin of the page, we have noted the location of the insertion or addition in our annotation.

10. The Editorial Notes preceding the Textual Notes contain translations of Latin passages, and the details of papers and books Reid quotes from or refers to in his texts. In many cases we have also referred to literature which we have no direct evidence that Reid used, but which we believe is a help to the reader in understanding which subjects Reid was addressing. Detailed commentary on the contents of the manuscripts has been confined to our editorial introduction.

The Editorial Notes are indicated in Reid's texts by asterisks * and are keyed to the texts using the same convention as that employed in the Textual Notes.

Where known, life dates for all figures active prior to 1800 mentioned in the notes to the Introduction and the Editorial Notes are given in the Index of Persons and Titles. 